\title{
Development of Non-bacterial Thrombotic Endocarditis after Percutaneous Transvenous Mitral Commissurotomy for Severely Calcified Mitral Stenosis
}

\author{
Shigehiro Tомімото, MD, Shigenori ITO, MD, Takahiko SuZUKI, ${ }^{1}$ MD, \\ Akira MishIMA, ${ }^{2}$ MD, Hiroshi SuZUMURA, MD, Yutaka TAKEDA, MD, \\ Yoshiaki YAMADA, MD, Toru HoRIO, MD, Akitomo GOTO, MD, \\ Shogo SUZUKI, MD, Tatsuya FUKUTOMI, MD, and Makoto ITOH, MD
}

\begin{abstract}
SUMMARY
We encountered a case of mitral stenosis, complicated with non-bacterial thrombotic endocarditis, that developed after percutaneous transvenous mitral commissurotomy (PTMC). A 71-year-old female Japanese patient had severe congestive heart failure and underwent PTMC for critical and severely calcified mitral stenosis. Four weeks later, the echocardiogram demonstrated a highly echoic protrusion in the postero-medial commissure of the mitral valve. There was little evidence of inflammation at that time. She had been anticoagulated adequately since she was admitted. The patient underwent replacement of the mitral valve. She did not show any evidence of systemic embolization. Microscopic evaluation showed only organized thrombus but no evidence of inflammation in the mitral valve. Silent development of non-bacterial thrombotic endocarditis after PTMC should be recognized as a rare but potentially lethal complication of PTMC. (Jpn Heart J 2000; 41: 411-416)
\end{abstract}

Key words: Complication, Balloon valvuloplasty, Vegetation

Percutaneous transvenous mitral commissurotomy (PTMC) is now an effective and safe treatment for selected patients with mitral stenosis. ${ }^{1-3)}$ With increasing performance of PTMC, the therapeutic effectiveness of this procedure has come to be widely accepted despite its risk. ${ }^{2,3)}$ Furthermore, an indication of PTMC has been extended to include even relatively complicated cases. To date, there have been no reports of the occurrence of non-bacterial thrombotic endocarditis (NBTE) ${ }^{4-10)}$ at the dilated site after this procedure. We report a case of thrombus formation that occurred insidiously as a rare complication of PTMC.

From the First Department of Internal Medicine, Nagoya City University Medical School, ${ }^{1}$ Division of Cardiology, Toyohashi Heart Center, ${ }^{2}$ First Department of Surgery, Nagoya City University, Medical School, Aichi, Japan.

Address for correspondence: Shigenori Ito, MD, the First Department of Internal Medicine, Nagoya City University Medical School, Kawasumi 1, Mizuho-cho, Mizuho-ku, Nagoya-shi, Aichi 467-8602, Japan.

Received for publication February 21, 2000.

Revised and accepted March 9, 2000. 


\section{CASE RePORT}

The patient was a 71-year-old Japanese female who presented at our hospital with severe dyspnea and peripheral edema on July 28, 1998. Three days before admission, a fever of $38^{\circ} \mathrm{C}$ had developed. The patient had a past history of cerebral infarction with right hemiplegia and motor aphasia. She had suffered from atrial fibrillation for the preceding six years. The patient was transferred directly to the intensive care unit of our hospital, where she was intubated and put under controlled ventilation with infusion of inotropic agents. She was found to have mitral stenosis with an estimated valvular area of $1.0 \mathrm{~cm}^{2}$, a mean pressure gradient of $12 \mathrm{mmHg}$ across the mitral valve and a cardiac index of $2.1 \mathrm{l} / \mathrm{min} / \mathrm{m}^{2}$ on echocardiography. The valve echo score ${ }^{3)}$ was 9 , with grade 4 calcification, grade 3 reduced leaflet mobility, and grade 2 leaflet thickening scores. She also had mild aortic stenosis, which we decided not to treat. Abnormal laboratory findings included a leukocyte count of $28,700 / \mathrm{mm}^{3}$ and a C-reactive protein concentration of $14.0 \mathrm{mg} / \mathrm{d} l$ (Figure 1). Although multiple blood cultures were negative, intravenous administration of ampicillin and panipenem was continued until PTMC, reducing the

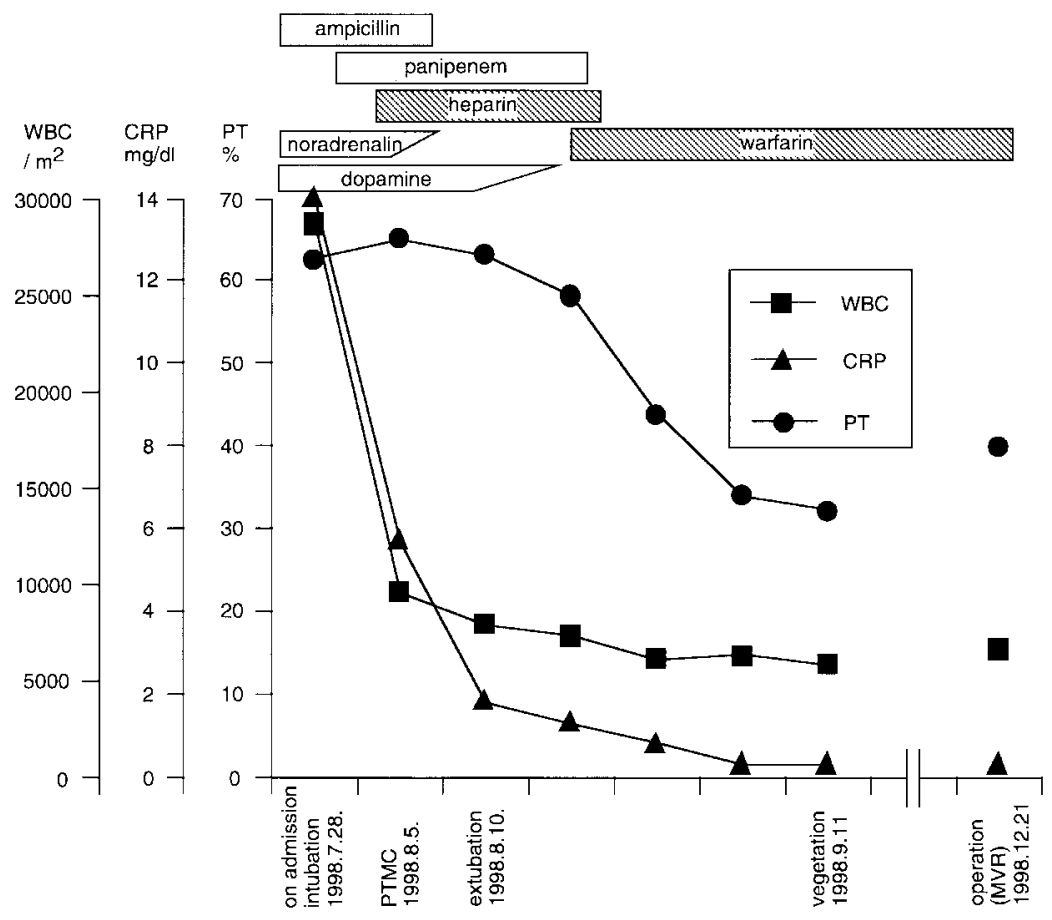

Figure 1. Clinical course and treatments. 
C-reactive protein concentration to $5.8 \mathrm{mg} / \mathrm{d} l$. She was inotrope-dependent with severe mitral valve stenosis since admission. Intensive pharmacological treatment did not improve her respiratory and hemodynamic condition, and she could not be weaned from a respirator. We therefore performed PTMC for mitral stenosis on August 5. We used an Inoue balloon and the balloon diameter was adjusted to $22 \mathrm{~mm}$ at the first inflation and $26 \mathrm{~mm}$ at the second. The result of PTMC was acceptable as a bailout alternative and technically uncomplicated. PTMC increased the mitral valvular area to $1.4 \mathrm{~cm}^{2}$ and decreased the mean transvalvular gradient to $6 \mathrm{mmHg}$. One week after PTMC, a chest roentgenogram revealed remarkable improvement of lung congestion, and she could be extubated. Intravenous administration of panipenem was continued until the serum level of C-reactive protein was normalized. Both the leukocyte count and serum level of C-reactive protein normalized after PTMC. However, four weeks after the valvuloplasty, two-dimensional echocardiography showed a highly echoic protrusion suggesting vegetation on the posterior commissure of the mitral valve (Figure 2). The vegetation was $10 \times 7 \mathrm{~mm}$ and fluctuated synchronously with the cardiac cycle. Anticoagulation by heparin infusion was started on the first day of admission. The prothrombin time was controlled at $60 \%$ before PTMC and $30 \%$ when vegetation was recognized. Although an immediate mltral value replacement was considered to be necessary, it was performed only about three months later on December 21 due to personal reasons. She was found to have a moderate degree of mitral leaflet thickening with some erosion on the free edge and the commissural fusion. However, the main body of the vegetation was

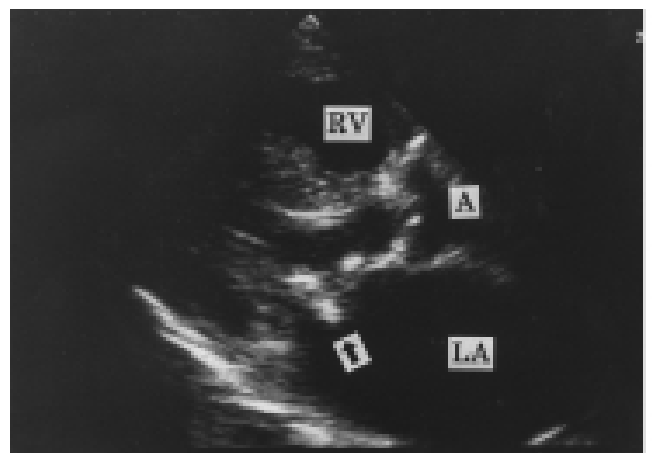

A

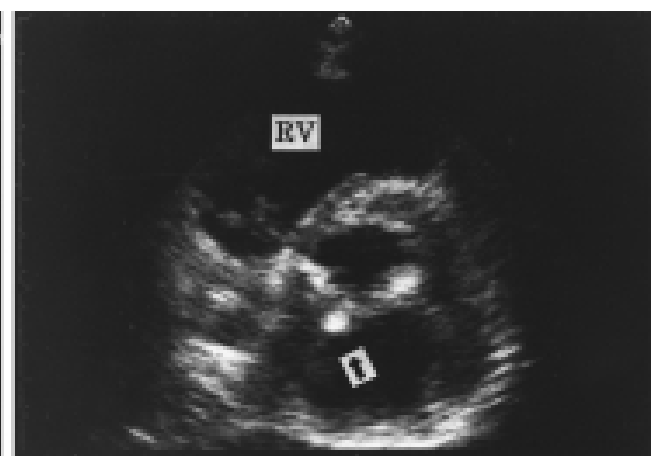

B

Figure 2. Two-dimensional echocardiographies demonstrate a highly echoic protrusion (arrows) indicating vegetation on the posterior commissure of the mitral valve four weeks after the valvuloplasty in parasternal long-axis (A) and short-axis views (B). The vegetation is $10 \times 7 \mathrm{~mm}$ and fluctuated synchronously with cardiac cycle in the left atrium. $\mathrm{A}=$ aorta; $\mathrm{LA}=$ left atrium; $\mathrm{RV}=$ right ventricle. 


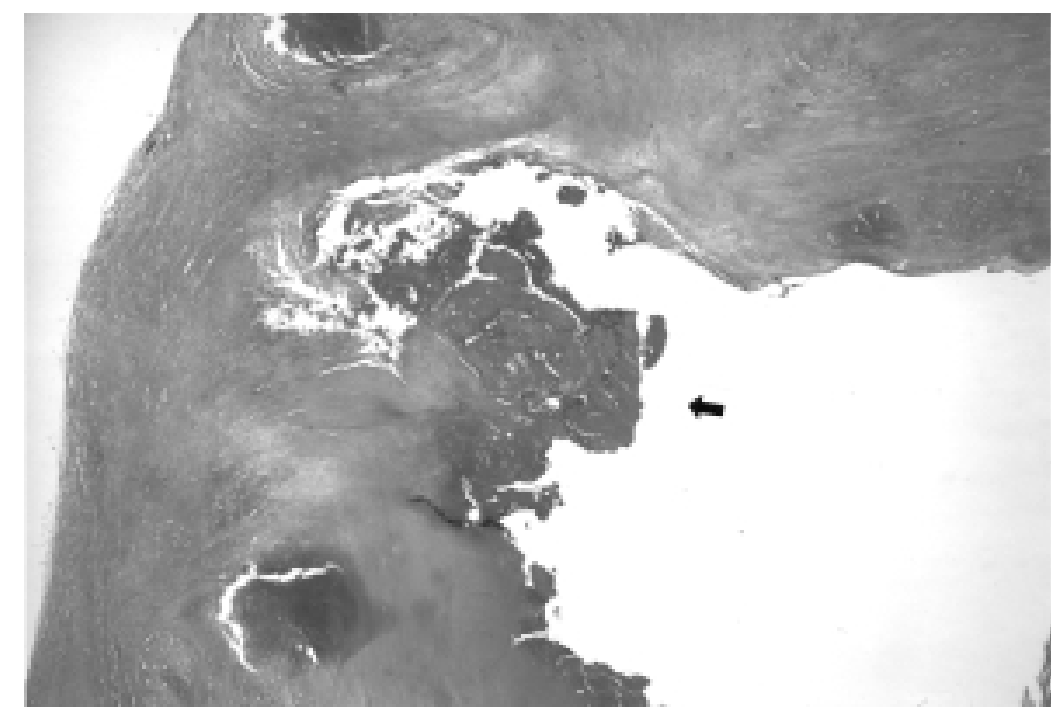

Figure 3. Low power photomicrograph of a mitral valve. Residual vegetation appears on the base of the dilated posterior commissure. It is compatible with organized thrombus with no inflammatory reaction found around the dilated lesion.

not found and only the basal portion of the vegetation was recognized on the posteromedial commissure at operation. She underwent annuloplasty of the tricuspid valve in addition to mitral valve replacement. Intraoperative culture was negative for microorganisms. The thrombus together with the valve were excised and sent to the pathology laboratory. Microscopically, the residual vegetation was compatible with organized thrombus with no inflammatory reaction (Figure 3). Follow-up echocardiography revealed no evidence of thrombus formation or perivalvular leakage.

\section{DISCUSSION}

We have reported a case of non-bacterial thrombotic endocarditis $(\mathrm{NBTE})^{4-10)}$ occurring as a rare complication of PTMC despite adequate anticoagulation therapy. The protrusion recognized in this case appeared to be an organized thrombus, based on its echocardiographic and microscopic features. One important possibility is that the protrusion was a vegetation which had developed from infective endocarditis. ${ }^{11-13)}$ Although we can not deny the possibility that the protrusion was a vegetation caused by infective endocarditis because echocardiographic findings were mimicking bacterial vegetation, the insidious clinical course, multiple sterile 
blood cultures, and macroscopic and microscopic findings support the diagnosis of NBTE. Furthermore, when we found the protrusion on the mitral valve, inflammation had already been almost controlled, as shown in Figure 1.

We do not know why the main body of the vegetation was eliminated at the time of operation. One possibility is that thrombolysis occurred as a result of intensive anticoagulation. The other possibility is that the vegetation became detached from the valve but fortunately did not result in distal embolization.

Although NBTE can no longer be considered a harmless or inconsequential incidental autopsy finding in elderly, terminally ill, or emaciated patients, ${ }^{14)}$ its antemortem diagnosis is difficult on clinical grounds and by non-invasive techniques because of the absence of pathognomonic symptoms and signs. ${ }^{7)}$ NBTE has been associated with a spectrum of underlying diseases. ${ }^{9}$ ) However, it is most commonly observed in patients with malignant neoplasms, ${ }^{9,14)}$ particularly adenocarcinoma, or disseminated intravascular coagulation. ${ }^{14,15}$ ) On the other hand, NBTE also develops in patients with chronic rheumatic valvulitis ${ }^{10)}$, or non-specific abnormalities such as fibrous thickening or fibrous and hyaline nodularitis of heart valves. Mechanical damage to valves may occur with catheters used for venous pressure ${ }^{4)}$ or pulmonary artery pressure monitoring ${ }^{8)}$, leading to the formation of verrucae. To the best of our knowledge, this is the first reported case of the occurrence of NBTE after valvular damage caused by PTMC.

As reported by Garrison, et al. ${ }^{5)}$ it is possible that endocardial damage induced by a catheter can cause adhesion of fibrin to the mitral valve with subsequent development of non-bacterial endocarditis; this may have been the etiology of the vegetation-like highly echoic mass in the present case. The therapeutic effectiveness of PTMC is based on mechanical fracturing of the fused commissure. Furthermore, the site of damage induced by balloon dilatation on an already compromised valve would be a suitable location for bacterial implantation. ${ }^{11-13,16)}$ In previous reports, patients with a high valve echo score appeared to be vulnerable to fibrin formation and subsequent thrombus formation or growth of vegetation. Despite the high valve echo score of 9, vegetation was not infected in this case.

Conclusion: Although NTBE after PTMC has not previously been reported, it should always be recognized as a possible complication, especially when PTMC is performed for mitral stenosis with a high valve echo score. Because NTBE may result in lethal systemic embolization, ${ }^{7,14}$ meticulous follow-up, including regular echocardiography, should be performed after 


\section{PTMC for mitral stenosis with unfavorable morphology.}

\section{REFERENCES}

1. Inoue K, Owaki T, Nakamura T, Kitamura F, Miyamoto N. Clinical application of transvenous mitral commissurotomy by a new balloon catheter. J Thorac Cardiovasc Surg 1984; 87: 394-402.

2. Iung B, Cormier B, Ducimetière P, et al. Immediate results of percutaneous mitral commissurotomy: a predictive model on a series of 1514 patients. Circulation 1996; 94: 2124-30.

3. Palacios IF, Block PC, Wilkins GT. Follow-up of patients undergoing percutaneous mitral balloon valvotomy: analysis of factors determining restenosis. Circulation 1989; 79: 573-9.

4. Becker AE, Becker MJ, Martin FH, Edwards JE. Bland thrombosis and infection in relation to intracardiac catheter. Circulation 1972; 46: 200-3.

5. Garrison PK, Freedman LR. Experimental endocarditis I: staphylococcal endocarditis in rabbits resulting from placement of a polyethylene catheter in the right side of the heart. Yale J Biol Med 1970; 42: 394-410.

6. Lopez JA, Fishbein MC, Siegel RJ. Echocardiographic features of nonbacterial thrombotic endocarditis. Am J Cardiol 1987; 59: 478-480.

7. Lopez JA, Ross RS, Fishbein MC, Siegel RJ. Nonbacterial thrombotic endocarditis: a review. Am Heart J 1987; 113: 773-84.

8. Pace NL, Horton W. Indwelling pulmonary artery catheters. Their relationship to aseptic thrombotic endocardial vegetations. JAMA 1975; 233: 893-4.

9. Waller BF, Knapp WS, Edwards JE. Marantic valvular vegetations. Circulation 1973; 48: 644-50.

10. Kardaras FG, Kardara DF, Rontogiani DP, et al. Acute aortic regurgitation caused by non-bacterial thrombotic endocarditis. Eur Heart J 1995; 16: 1152-4.

11. Inoue M, Sato S, Kato H, Sakagoshi N, Inoue T, Nagai I. A case of infective mitral endocarditis after percutaneous transvenous mitral commissurotomy. J Jpn Assn Thorac Surg 1995; 43: 493-6 (in Japanese).

12. Kulkarni SM, Loya YS, Sharma S. Infective endocarditis following balloon dilatation of mitral valve. Int J Cardiol 1992; 34: 103-5.

13. Moriyama Y, Toyohira H, Saigenji H, Shimokawa S, Taira A. Infective mitral valve endocarditis after percutaneous transvenous mitral commissurotomy. Eur J Cardiothorac Surg 1995; 9: 111-2.

14. Deppisch LM, Fayemi AO. Non-bacterial thrombotic endocarditis. Clinicopathologic correlations. Am Heart J 1976; 92: 723-9.

15. Sugiura M, Hiraoka K, Ohkawa SI, Veda K, Matsuda T, Murakami A. A clinicopathological study on cardiac lesions in 64 cases of disseminated intravascular coagulation. Jpn Heart J 1977; 18: 57-69.

16. Tada Y, Sato N, Fujioka M, et al. A case of mitral stenosis developing infective endocarditis 18 months after PTMC. Kokyu to Junkan 1991; 39: 725-8 (in Japanese). 
